\title{
РАСЧЕТ ИНТЕГРАЛЬНЫХ ХАРАКТЕРИСТИК ЛИНЕЙНОЙ АСИНХРОННОЙ МАШИНЫ С УЧЕТОМ ПРОДОЛЬНОГО КРАЕВОГО ЭФФЕКТА И КОНЕЧНОЙ ШИРИНЫ
}

В $\left.{ }^{1}{ }^{1}\right]$ на основании двумерной расчетной модели (рис. 1) определены аналитические выражения для напряженности магнитного поля в активной части воздушного зазора линейной асинхронной машины (ЛАМ). Положив в них в случае однослойной обмотки с полным шагом $\beta=1$ и $g=0$, получим
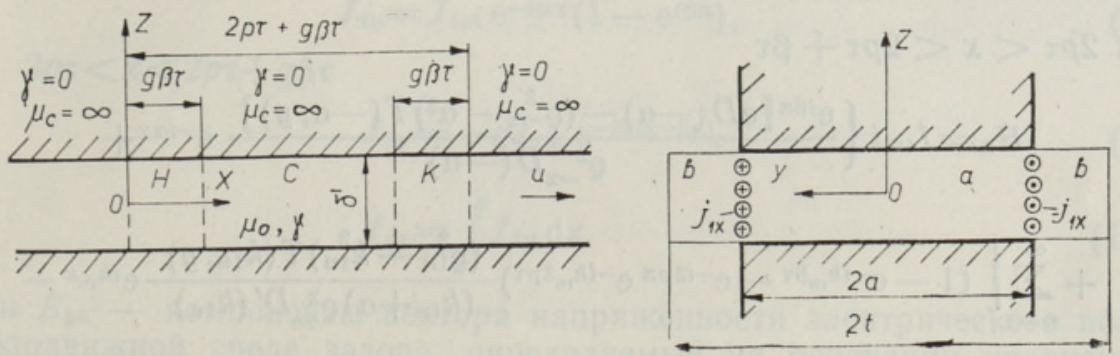

Рис. 1. Упрощенная расчетная модель ЛАМ.

$$
\begin{aligned}
& \dot{H}_{\mathrm{a}}= 2 J_{1 m} i\left\{\frac{\left(\varrho_{-\alpha}^{2}-\alpha^{2}\right) T(-\alpha, y)-\alpha D(-\alpha)}{\varrho_{-\alpha}^{2} D(-\alpha)} \mathrm{e}^{-i \alpha x}+\right. \\
&+\sum_{n=1}^{\infty}\left[\frac{\left(\varrho_{1 n}^{2}-k_{1 n}^{2}\right) T\left(k_{1 n}, y\right)}{\left(k_{1 n}+\alpha\right) \varrho_{1 n}^{2} D^{\prime}\left(k_{1 n}\right)} \mathrm{e}^{i k_{1 n} x}+T\left(k_{2 n}, y\right) \mathrm{e}^{i k_{2 n}(x-2 p \tau)} \times\right. \\
&\left.\left.\times \frac{\varrho_{2 n}^{2}-k_{2 n}^{2}}{\left(k_{2 n}+\alpha\right) \varrho_{2 n}^{2} D^{\prime}\left(k_{2 n}\right)}\right]+\frac{\operatorname{ch}\left(\alpha \sqrt{i \varepsilon_{0}} \zeta\right)}{\alpha D^{\prime}(0)}\right\} .
\end{aligned}
$$

Если обмотка индуктора ЛАМ двуслойная с укороченным шагом $g=1$ и $\beta \neq 1$, то при $0<x<\beta \tau$

$$
\dot{H}_{\mathrm{a}}=J_{1 m} i\left\{\frac{\left(\mathrm{Q}_{-\alpha}^{2}-\alpha^{2}\right) T(-\alpha, y)-\alpha D(-\alpha)}{\mathrm{Q}_{-\alpha}^{2} D(-\alpha)} \mathrm{e}^{-i \alpha x}+\right.
$$




$$
\begin{gathered}
+\sum_{n=1}^{\infty}\left[\frac{\left(\varrho_{1 n}^{2}-k_{1 n}^{2}\right) T\left(k_{1 n}, y\right)}{\left(k_{1 n}+\alpha\right) \varrho_{1 n}^{2} D^{\prime}\left(k_{1 n}\right)} \mathrm{e}^{i k_{1 n} x}+\left(\mathrm{e}^{-i k_{2 n} \beta \tau}+\mathrm{e}^{-i 2 p \pi} \mathrm{e}^{-i k_{2 n} 2 p \tau}-\right.\right. \\
\left.\left.-\mathrm{e}^{-i 2 p \pi} \mathrm{e}^{-i k_{2 n}(2 p \tau+\beta \tau)}\right) \frac{\left(\varrho_{2 n}^{2}-k_{2 n}^{2}\right) T\left(k_{2 n}, y\right)}{\left(k_{2 n}+\alpha\right) \varrho_{2 n}^{2} D^{\prime}\left(k_{2 n}\right)} \mathrm{e}^{i k_{2 n} x}\right]+ \\
\left.+\frac{\operatorname{ch}\left(\alpha \sqrt{i \varepsilon_{0}} y\right)}{\alpha D^{\prime}(0)}\right\},
\end{gathered}
$$

при $\beta \tau<x<2 p \tau$

$$
\begin{gathered}
\dot{H}_{\mathrm{a}}=J_{1 m} i\left\{\frac{\left(1-\mathrm{e}^{i \beta \pi}\right)\left[\left(\varrho_{-\alpha}^{2}-\alpha^{2}\right) T(-\alpha, y)-\alpha D(-\alpha)\right]}{\varrho_{-\alpha}^{2} D(-\alpha)} \mathrm{e}^{-i \alpha x}+\right. \\
+\sum_{n=1}^{\infty}\left[\frac{\left(1-\mathrm{e}^{-i k_{1 n} \beta \tau}\right)\left(\varrho_{1 n}^{2}-k_{1 n}^{2}\right) T\left(k_{1 n}, y\right)}{\left(k_{1 n}+\alpha\right) \mathrm{Q}_{1 n}^{2} D^{\prime}\left(k_{1 n}\right)} \mathrm{e}^{i k_{1 n} x}+\left(\mathrm{e}^{-i 2 p \pi} \mathrm{e}^{-i k_{2 n} 2 p \tau}-\right.\right. \\
\left.\left.\left.-\mathrm{e}^{-i 2 p \pi} \mathrm{e}^{-i k_{2 n}(2 p \tau+\beta \tau)}\right) \frac{\left(\varrho_{2 n}^{2}-k_{2 n}^{2}\right) T\left(k_{2 n}, y\right)}{\left(k_{2 n}+\alpha\right) \varrho_{2 n}^{2} D^{\prime}\left(k_{2 n}\right)} \mathrm{e}^{i k_{2 n} x}\right]\right\},
\end{gathered}
$$

при $2 p \tau<x<2 p \tau+\beta \tau$

$$
\dot{H}_{\mathrm{a}}=J_{1 m} i\left\{\frac{\mathrm{e}^{i \beta \pi}\left[\alpha D(-\alpha)-\left(\varrho_{-\alpha}^{2}-\alpha^{2}\right) T(-\alpha, y)\right]}{\varrho_{-\alpha}^{2} D(-\alpha)} \mathrm{e}^{-i \alpha x}+\right.
$$$$
+\sum_{n=1}^{\infty}\left[\left(1-\mathrm{e}^{-i k_{1 n} \beta \tau}-\mathrm{e}^{-i 2 p \pi} \mathrm{e}^{-i k_{1 n} 2 p \tau}\right) \frac{\left(\varrho_{1 n}^{2}-k_{1 n}^{2}\right) T\left(k_{1 n}, y\right)}{\left(k_{1 n}+\alpha\right) \varrho_{1 n}^{2} D^{\prime}\left(k_{1 n}\right)} \mathrm{e}^{i k_{1 n} x}-\right.
$$

$\left.\left.-\mathrm{e}^{-i 2 p \pi} \mathrm{e}^{-i k_{2 n}(2 p \tau+\beta \tau)} \frac{\left(\varrho_{2 n}^{2}-k_{2 n}^{2}\right) T\left(k_{2 n}, y\right)}{\left(k_{2 n}+\alpha\right) \varrho_{2 n}^{2} D^{\prime}\left(k_{2 n}\right)} \mathrm{e}^{i k_{2 n} x}\right]-\mathrm{e}^{-i 2 p \pi} \frac{\operatorname{ch}\left(\alpha \overline{\gamma i \varepsilon_{0}} y\right)}{\alpha D^{\prime}(0)}\right\}$.

Здесь $f_{1 m}$ - комплексная амплитуда плотности первичного тока,

$$
\begin{aligned}
& D(k)=\varrho \operatorname{sh} \varrho a \operatorname{sh} k(t-a)+k \operatorname{ch} \varrho a \operatorname{ch} k(t-a), \\
& D^{\prime}(k)=\partial D(k) / \partial k, \\
& T(k, y)=\operatorname{ch} k(t-a) \operatorname{ch} \varrho y, \\
& \varrho^{2}=k^{2}+i \mu_{0} \gamma u k+i \alpha^{2} \varepsilon_{0},
\end{aligned}
$$

$\varepsilon_{0}=\mu_{0} \gamma \omega / \alpha^{2}, \alpha=\frac{\pi}{\tau}, \varepsilon=\varepsilon_{0} s, s-$ скольжение, $\gamma-$ расчетная проводимость вторичной шины, $u-$ скорость ее движения, $\omega-$ круговая частота, $\tau$ - полюсное деление, $p$ - число пар полюсов, $\beta$ - относительный шаг обмотки; $k_{1 n}, k_{2 n}-n$-я пара ненулевых корней уравнения $D(k)=0, \varrho_{1 n^{2}}$ и $\varrho_{2 n^{2}}{ }^{2}-$ значения $\mathrm{Q}^{2}$ при $k=k_{1 n}$ и $k=k_{2 n}$ соответственно.

В выражениях (1)-(4) член, содержаший $\mathrm{e}^{-i \alpha x}$, представляет собой 
нормальную бегущую волну, члены с $\mathrm{e}^{i k_{n} x}, \mathrm{e}^{i k 2 n x}-$ волны продольного краевого эффекта и последний член - пульсирующую составляющую напряженности магнитного поля.

\section{Электромагнитная мощность. Параметры схемы замещения}

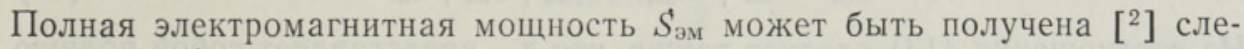
дующим образом:

$$
S_{\text {วМ }}=-\frac{1}{2} \delta^{\prime}\left(\int_{-a}^{a} \mathrm{~d} y \int_{0}^{2 p \tau+g \beta \tau} \dot{E}_{y \mathrm{a}} \dot{J}_{1 y} \mathrm{~d} x+\left.2 \int_{0}^{2 p \tau+g \beta \tau} \dot{E}_{x \mathrm{a}}\right|_{y=\mathrm{a}} \dot{J}_{1 x} \mathrm{~d} x\right)
$$

Здесь, в силу принятых допущений, интегрирование по $\mathrm{d} z$ сводится к умножению на величину приведенного зазора $\delta^{\prime}$.

В выражении (6) $\dot{J}_{1 y}$ и $\dot{J}_{1 x}-$ сопряженные комплексы плотностей первичного тока и тока лобовых частей обмотки соответственно. Согласно $\left[{ }^{1}\right]$,

при $0<x<g \beta \tau$

$$
J_{1 y}=g f_{1 m} \mathrm{e}^{-i \alpha x},
$$

при $\quad g \beta \tau<x<2 p \tau$

$$
J_{1 y}=J_{1 m} \mathrm{e}^{-i \alpha x}\left(1-\mathrm{e}^{i \beta \pi}\right),
$$

при $2 p \tau<x<2 p \tau+g \beta \tau$

a

$$
\begin{aligned}
& J_{1 y}=-g f_{1 m} \mathrm{e}^{-i(\alpha x-\beta \pi),} \\
& J_{1 x}=\int_{0}^{x} J_{1 y} \mathrm{~d} x .
\end{aligned}
$$

$\dot{E}_{x a}$ и $\dot{E}_{y \text { a }}$ - компоненты вектора напряженности электрического поля в неподвижной среде зазора, определяемые на основании уравнений Максвелла $\left[{ }^{2}\right]$ с учетом того, что $\dot{H}=\dot{H}_{z}$ и $u=u_{x}$ :

$$
\begin{aligned}
& \dot{E}_{x \mathrm{a}}=\frac{1}{\gamma} \frac{\partial \dot{H}_{\mathrm{a}}}{\partial y}, \\
& \dot{E}_{y \mathrm{a}}=-\frac{1}{\gamma}\left(\frac{\partial \dot{H}_{\mathrm{a}}}{\partial x}+J_{\mathrm{cm}}\right)+u \mu_{0} \dot{H}_{\mathrm{a}} .
\end{aligned}
$$

Анализ работы ЛАМ целесообразно проводить на основе последовательной схемы замещения $\left[{ }^{3}\right]$. Расчет сопротивлений схемы замещения (рис. 2) удобно выполнять в относительных единицах, принимая за базисное $x_{\Gamma 1}$ - главное индуктивное сопротивление обмотки индуктора $\left[{ }^{2}\right]$.

Рис. 2. Схема замещения ЛАМ с учетом краевых эффектов.

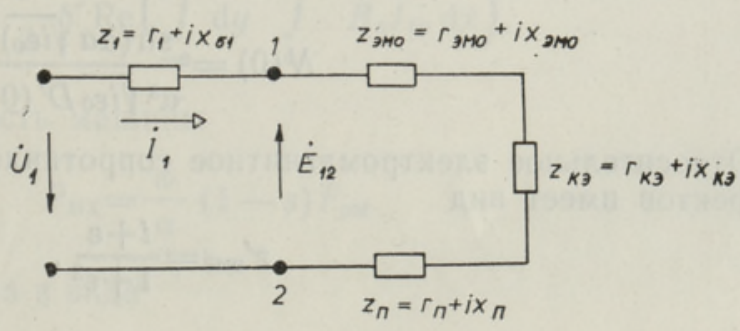


Относительное сопротивление схемы замещения $z_{\text {әм}}$, определяющее полную электромагнитную мощность ЛАМ, можно получить из выражения (6), преобразовав его к виду

причем

$$
S_{\text {әм }}=m I_{1}^{2} x_{\mathrm{T} 1} z_{\text {әм }},
$$

$$
z_{\text {әм }}=r_{\text {эм }}+i x_{\text {әм }}=z_{\text {әм } 0}+z_{\text {кә }}+z_{\text {II }}
$$

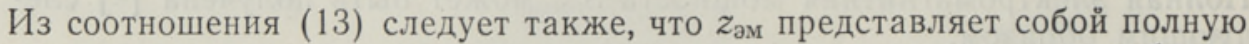
электромагнитную мощность в относительных единицах, если за базисную величину принято

$$
S_{\text {วмб }}=m I_{1}^{2} x_{\mathrm{r} 1}=\frac{1}{2} \delta^{\prime} \frac{4 J_{1 m}^{2}}{\alpha^{2}} \mu_{0} \omega 2 a p \tau \sin ^{2} \frac{\beta \pi}{2} .
$$

Для машины с однослойной обмоткой и четным числом полюсов (при $\beta=1$ и $g=0$ ) относительное сопротивление от нормальной бегущей волны магнитного поля с учетом поперечного краевого эффекта на основании зависимостей $(1),(6),(8),(10)-(14)$ равно

$$
z_{\text {วм } 0}=\frac{i+\varepsilon}{1+\varepsilon^{2}}\left(1-i \varepsilon \frac{\alpha}{a} N(-\alpha)\right) .
$$

Относительное сопротивление от полей продольного краевого эффекта с учетом влияния конечной ширины машины:

$$
\begin{gathered}
z_{\text {кə }}=\frac{1}{2 p \tau a}\left[\sum_{n=1}^{\infty} \frac{\alpha^{2}}{k_{1 n}} N\left(k_{1 n}\right)\left(\varrho_{1 n}^{2}-k_{1 n}^{2}\right)\left(\mathrm{e}^{i k_{1 n} 2 p \tau}-1\right)+\right. \\
+\frac{\alpha^{2}}{k_{2 n}} N\left(k_{2 n}\right)\left(\mathrm{Q}_{2 n}^{2}-k_{2 n}^{2}\right)\left(1-\mathrm{e}^{\left.-i k_{2 n} 2 p \tau\right)}\right] .
\end{gathered}
$$

Относительное сопротивление от пульсирующей составляющей поля:

$$
z_{\mathrm{I}}=i \frac{\alpha^{2}}{a} N(0)
$$

где

$$
\begin{gathered}
N\left(k_{1 n}\right)=\frac{\operatorname{ch} k_{1 n}(t-a) \operatorname{sh} \varrho_{1 n} a}{\left(k_{1 n}+\alpha\right)^{2} \varrho_{1 n}^{3} D^{\prime}\left(k_{1 n}\right)}, \\
N\left(k_{2 n}\right)=\frac{\operatorname{ch} k_{2 n}(t-a) \operatorname{sh} \varrho_{2 n} a}{\left(k_{2 n}+\alpha\right)^{2} \varrho_{2 n}^{3} D^{\prime}\left(k_{2 n}\right)}, \\
N(-\alpha)=\frac{\operatorname{ch~} \alpha(a-t) \operatorname{sh}(\alpha a \sqrt{1+i \varepsilon})}{\alpha \sqrt{1+i \varepsilon} D(-\alpha)}, \\
N(0)=\frac{\operatorname{sh}\left(\alpha a \sqrt{i \varepsilon_{0}}\right)}{\alpha^{3} \sqrt{i \varepsilon_{0}} D^{\prime}(0)} .
\end{gathered}
$$

Относительное электромагнитное сопротивление без учета краевых эффектов имеет вид

$$
z_{\text {әм } 0}^{\prime}=\frac{i+\varepsilon}{1+\varepsilon^{2}} .
$$


В случае двуслойной обмотки индуктора с наполовину заполненными пазами концевых зон (при $\beta \neq 1, g=1$ ) относительные электромагнитные сопротивления определяются аналогичным образом с учетом соотношений $(2)-(4),(6)$ и $(7)-(14)$

$$
\begin{aligned}
& z_{\text {әм } 0}=\frac{i+\varepsilon}{1+\varepsilon^{2}}\left[\left(1+\frac{\beta \tau}{2 p \tau} \frac{\cos \beta \pi}{2 \sin ^{2} \frac{\beta \pi}{2}}\right)\left(1-i \varepsilon \frac{\alpha}{a} N(-\alpha)\right)\right]+ \\
& +i \frac{s}{2 p \tau a} N(-\alpha) \operatorname{ctg} \frac{\beta \pi}{2}, \\
& z_{\mathrm{k} \vartheta}=\frac{1}{2 p \tau a} \sum_{n=1}^{\infty}\left\{N ( k _ { 1 n } ) ( \varrho _ { 1 n } ^ { 2 } - k _ { 1 n } ^ { 2 } ) \left[\frac { \alpha ^ { 2 } } { k _ { 1 n } } \left(\frac{\mathrm{e}^{i k_{\mathrm{in}} \beta \tau}-1}{2 \sin ^{2} \frac{\beta \pi}{2}}+\right.\right.\right. \\
& \left.+\mathrm{e}^{i 2 p \pi} \mathrm{e}^{i k_{1 n} 2 p \tau} \frac{\sin ^{2} \frac{k_{1 n} \beta \tau}{2}}{\sin ^{2} \cdot \frac{\beta \pi}{2}}\right)-\operatorname{ctg} \frac{\beta \pi}{2}\left(\frac{k_{1 n}}{\varepsilon_{0}}\left(1+\frac{k_{1 n}}{\alpha}\right)+\right. \\
& \left.\left.+i(1-s) k_{1 n}+i \alpha(2-s)\right)\right]+N\left(k_{2 n}\right)\left(\varrho_{2 n}^{2}-k_{2 n}^{2}\right)\left[\frac { \alpha ^ { 2 } } { k _ { 2 n } } \left(\frac{1-\mathrm{e}^{-i k_{2 n} \beta \tau}}{2 \sin ^{2} \cdot \frac{\beta \pi}{2}}-\right.\right. \\
& \left.-\mathrm{e}^{-i 2 p \pi} \mathrm{e}^{-i k_{2 n} 2 p \tau} \frac{\sin ^{2} \frac{k_{2 n} \beta \tau}{2}}{\sin ^{2} \frac{\beta \pi}{2}}\right)-\operatorname{ctg} \frac{\beta \pi}{2}\left(\frac{k_{2 n}}{\varepsilon_{0}}\left(1+\frac{k_{2 n}}{\alpha}\right)+\right. \\
& \left.\left.\left.+i(1-s) k_{2 n}+i \alpha(2-s)\right)\right]\right\} \text {, } \\
& z_{\mathrm{I}}=i \frac{\alpha^{2}}{2 p \tau a} N(0)\left[\frac{\beta \tau}{2 \sin ^{2} \cdot \frac{\beta \pi}{2}}+\frac{s-2}{\alpha} \operatorname{ctg} \cdot \frac{\beta \pi}{2}\right] .
\end{aligned}
$$

\section{Электромагнитная сила. Механическая мощность}

Для получения электромагнитной силы, действующей на вторичную шину в направлении оси $x$, можно проинтегрировать силу, действующую на элемент объема первичной обмотки, по всему объему активной зоны и взять результат с противоположным знаком $\left[{ }^{2}\right]$ :

$$
F_{\text {эм }}=-\frac{1}{2} \delta^{\prime} \operatorname{Re}\left(\int_{-a}^{a} \mathrm{~d} y \int_{0}^{2 p \tau+g \beta \tau} \dot{B}_{\mathrm{a}} \dot{J}_{1 y} \mathrm{~d} x\right) .
$$

Механическая мощность машины

$$
P_{\mathrm{Mx}}=\frac{\omega}{\alpha}(1-s) F_{\text {эм }}
$$




$$
P_{\mathrm{Mx}}=(1-s) m I_{1}^{2} x_{\mathrm{r} 1} r_{F} .
$$

Параметр $r_{F}$, определяющий механическую характеристику машины, для ЛАМ с однослойной обмоткой (при $\beta=1$ и $g=0$ ) находим на основании выражений $(21)-(23)$ с учетом (1) и (8):

$$
\begin{gathered}
r_{F}=r_{\partial \mathrm{M} 0}^{F}+r_{\mathrm{K} F}=\operatorname{Re}\left\{\frac{i+\varepsilon}{1+\varepsilon^{2}}\left(1-i \varepsilon \frac{\alpha}{a} N(-\alpha)\right)-\frac{\alpha}{2 p \tau a} \sum_{n=1}^{\infty}\left[N ( k _ { 1 n } ) \left(\varrho_{1 n}^{2}-\right.\right.\right. \\
\left.\left.\left.-k_{1 n}^{2}\right)\left(\mathrm{e}^{i k_{1 n} 2 p \tau}-1\right)+N\left(k_{2 n}\right)\left(\varrho_{2 n}^{2}-k_{2 n}^{2}\right)\left(1-\mathrm{e}^{-i k_{2 n} 2 p \tau}\right)\right]\right\} .
\end{gathered}
$$

В случае двуслойной обмотки (при $\beta \neq 1$ и $g=1) r_{F}$ определяется из зависимостей $(21)-(23),(2)-(4)$ и $(7)-(9)$ :

$$
\begin{aligned}
r_{F}= & r_{\text {วм} 0}^{F}+r_{\mathrm{KF}}+r_{\Pi F}=\operatorname{Re}\left[\frac{i+\varepsilon}{1+\varepsilon^{2}}\left(1+\frac{\beta \tau}{2 p \tau} \frac{\cos \beta \pi}{2 \sin ^{2} \frac{\beta \pi}{2}}\right)\left(1-i \varepsilon \frac{\alpha}{a} N(-\alpha)\right)\right]- \\
& -\frac{\alpha}{2 p \tau a} \operatorname{Re}\left\{\sum _ { n = 1 } ^ { \infty } \left[N ( k _ { 1 n } ) ( \mathrm { Q } _ { 1 n } ^ { 2 } - k _ { 1 n } ^ { 2 } ) \left(\frac{\mathrm{e}^{i k n \beta \tau}-1}{2 \sin ^{2} \frac{\beta \pi}{2}}+\mathrm{e}^{i k_{1 n}{ }^{2 p \tau}} \mathrm{e}^{i 2 p \pi} \times\right.\right.\right. \\
& \left.\times \frac{\sin ^{2} \frac{k_{1 n} \beta \tau}{2}}{\sin ^{2} \frac{\beta \pi}{2}}+i \operatorname{ctg} \frac{\beta \pi}{2}\right)+N\left(k_{2 n}\right)\left(\varrho_{2 n}^{2}-k_{2 n}^{2}\right)\left(\frac{1-\mathrm{e}^{-i k_{2 n} \beta \tau}}{2 \sin ^{2} \frac{\beta \pi}{2}}-\right. \\
& \left.\left.\left.-\mathrm{e}^{-i k_{2 n} 2 p \tau} \mathrm{e}^{-i 2 p \pi} \frac{\sin ^{2} \frac{k_{2 n} \beta \tau}{2}}{\sin ^{2} \frac{\beta \pi}{2}}+i \operatorname{ctg} \frac{\beta \pi}{2}\right)\right]+i N(0) \operatorname{ctg} \frac{\beta \pi}{2}\right\} .
\end{aligned}
$$

В выражениях (24), (25) при $\beta=1$ имеем $r_{\text {әм0 }}^{F}=r_{\text {әм0 }}$ и $r_{\text {пF }}=0$.

\section{Анализ результатов}

Для оценки влияния поперечного и продольного краевых эффектов на электромагнитные и механические характеристики ЛАМ на основании полученных зависимостей были выполнены численные расчеты относительных величин сопротивлений схемы замещения и параметра $r_{F}$. При этом ненулевые корни трансцендентного уравнения

$$
D(k)=0
$$

вычислялись на ЭВМ обобщенным методом Ньютона $\left[{ }^{4}\right]$.

Определяющими при вычислении параметров краевого эффекта яв-

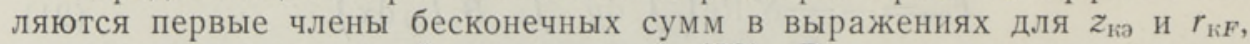
соответствующие первой паре корней (26). Вклад в решение членов, соответствующих последующим корням, зависит от величины добротности машины $\varepsilon_{0}$, числа пар полюсов $p$ и скольжения $s$. Так, для ЛАМ с малыми $\varepsilon_{0}\left(\varepsilon_{0}<5\right)$ пренебрежение второй парой корней при определении электромагнитных сопротивлений $r_{\text {эм и }} x_{\text {эм }}$ дает погрешность не более $0,1 \%$ вне зависимости от числа $p$. Однако с ростом $\varepsilon_{0}$ эта погрешность увеличивается и тем значительнее, чем меньше $p$. Например, при 


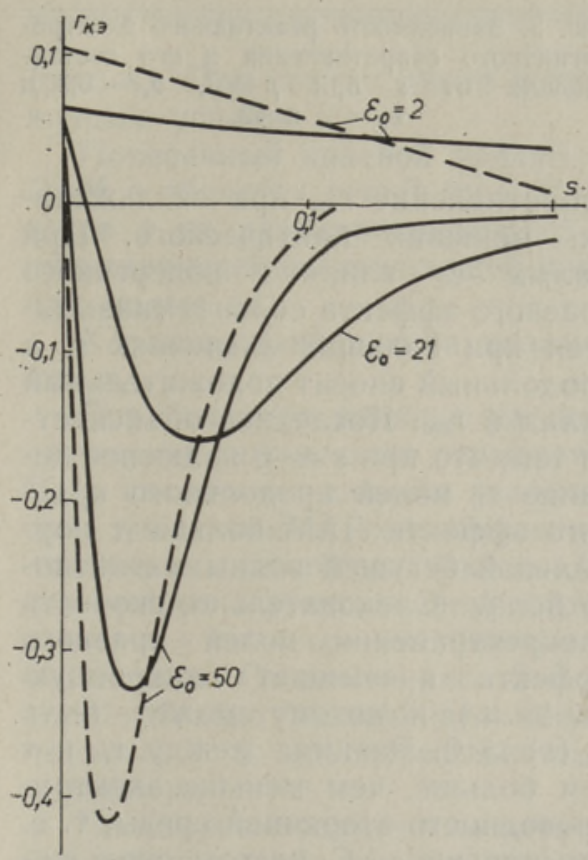

Рис. 3. Зависимость активного электромагнитного сопротивления краевого эффекта от $s$ и $\varepsilon_{0}$ при $p=6, b_{*}=$ $=(t-a) / \tau=0,3$. Расчет по двумерной (—) и одномерной (-.) теорин.

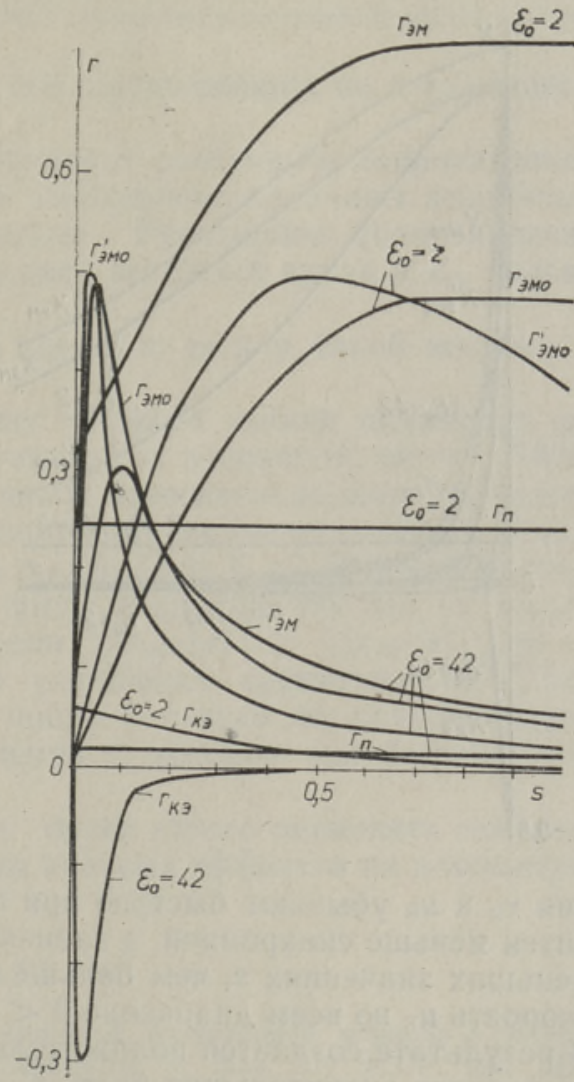

Рис. 4. Зависимость полного активного электромагнитного сопротивления и его составляющих от $s$ при $p=6, \quad b_{*}=0,3$ и $\varepsilon_{0}=2$ и 42.

$\varepsilon_{0}=40$ и $p=2$ она достигает $3 \%$, но снижается до $0,5 \%$ при $p=8$. При расчете механических характеристик $r_{F}(s)$ рассматриваемая погрешность больше, однако при $p=8$ в диапазоне $\varepsilon_{0} \leqslant 80$ не превышает $1,4 \%$. Пренебрежение остальными корнями уравнения (26) вносит в результаты вычислений изменения не более $0,5 \%$ во всех исследованных случаях. Для уменьшения влияния продольного краевого эффекта современные мощные ЛАМ создаются с большим числом полюсов. Поэтому в расчетах достаточно учитывать лишь первые члены бесконечных сумм $(n=1)$ в соотношениях (1)-(4), (16), (19), (24) и (25).

Анализ зависимости сопротивлений схемы замещения от $s$ и $\varepsilon_{0}$ для ЛАМ с однослойной обмоткой и четным числом полюсов показал, что поперечный краевой эффект, заключающийся в наличии $x$-составляющих вторичного тока $\left[{ }^{2}\right]$, способен существенно изменять характеристики машины. Сопротивление вторичной среды увеличивается, зависимости $r_{\text {әм0 }}=f(s)$ и $r_{\text {кә }}=f(s)$, учитывающие конечную ширину машины, становятся «мягче» зависимостей $r_{\text {әмо }}^{\prime}=f(s)$ и $r_{\text {кә }}^{\prime}=f(s)$, не учитывающих этого фактора (рис. 3,4$)$. Максимумы рассматриваемых функций сдвигаются в сторону больших скольжений и уменьшаются по абсолютной величине. Поперечный и продольный краевые эффекты в значительной степени уменьшают суммарное активное электромагнитное 


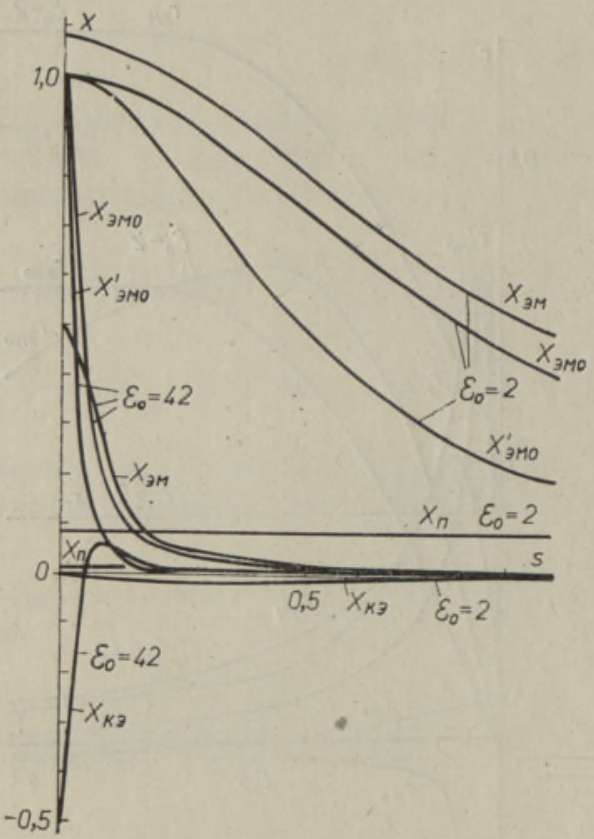

Рис. 5. Зависимость реактивного электромагнитного сопротивления и его составляющих от $s$ при $p=6, \quad b_{*}=0,3$ и $\varepsilon_{0}=2$ и 42.

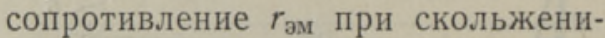
ях, меньших критического. При малых $\varepsilon_{0}$ влияние поперечного краевого эффекта сильнее сказывается при больших значениях $s$, а продольный вносит положительный вклад в $r_{\text {әм. }}$ Последнее объясняется тем, что при $s=0$ полюсное деление $\tau_{\mathrm{k}}$ полей продольного краевого эффекта ЛАМ больше $\tau$ нормальной бегущей волны магнитного поля. Следовательно, скорость распространения полей краевого эффекта превышает синхронную $\left(u_{\mathrm{k}}>u_{1}\right)$ и потому может быть $r_{\text {кә }}(s)>0$. Разница между $\tau_{\mathrm{k}}$ и $\tau$ тем больше, чем меньше электропроводность вторичной среды, т. е. -чем меньше $\varepsilon_{0}$. С ростом скольжения $\tau_{\text {к }}$ и $u_{\mathrm{k}}$ убывают быстрее при болыших $\varepsilon_{0}$, вследствие чего $u_{\mathrm{k}}$ становится меньше синхронной, а зависимость $r_{\text {кә }}(s)$ - отрицательной при тем меньших значениях $s$, чем больше $\varepsilon_{0}$ (рис. 3 ). При достаточно малых $\varepsilon_{0}$ скорость $u_{\mathrm{k}}$ во всем диапазоне $0<s<1$ может превышать синхронную. В результате создается положительное тяговое усилие. Сопротивление $r_{\text {I }}$

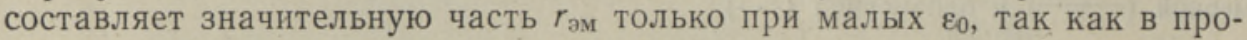
тивном случае пульсирующая составляющая первичного поля почти полностью демпфируется полем токов, наведенных во вторичной шине
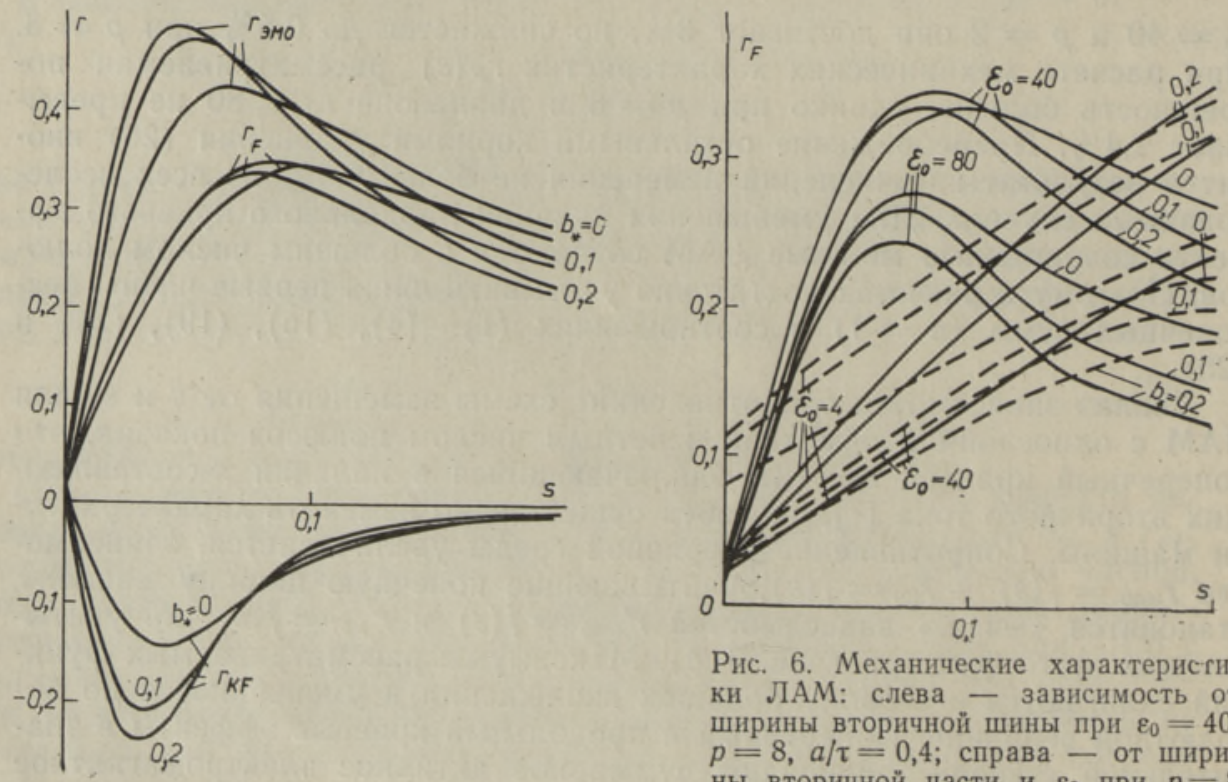

Рис. 6. Механические характерисгики ЛАМ: слева - зависимость от ширины вторичной шины при $\varepsilon_{0}=40$, $p=8, a / \tau=0,4$; справа - от ширины вторичной части и $\varepsilon_{0}$ при $p=8$ $(\longrightarrow)$ и 2 (- - $), a / \tau=0,4$. 
(рис. 4). При четном числе полюсов эта составляющая не создает движущего усилия.

Поперечный краевой эффект увеличивает реактивное сопротивление ЛАМ в областях $s \neq 0$ (рис. 5) из-за уменьшения величины вторичных токов и их размагничивающего действия. Реактивное сопротивление

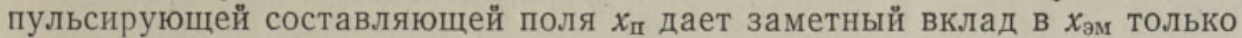
при малых $\varepsilon_{0}$.

Характер зависимости параметра $r_{\text {кF }}$ от $s, \varepsilon_{0}$ и $p$ такой же, как и для $r_{\text {кэ. }}$

Определенное влияние на величину тягового усилия оказывает соотношение поперечных размеров первичной и вторичной частей ЛАМ. При увеличении ширины вторичной шины относительно ширины индуктора уменьшается сопротивление вторичной среды, становятся более «жесткими» характеристики $r_{\text {эм0 }}(s)$ и $r_{\text {кF }}(s)$ (рис. $\left.6, a\right)$. Изменение этих параметров с уменьшением ширины шины различно, так как $\tau_{r}$ варьируют, а $\tau$ остается постоянным, и если $\left|\Delta r_{\text {әмо }}(s)\right|>\left|\Delta r_{\mathrm{KF}}(s)\right|$, то $r_{F}$ возрастает. Согласно рис. 6,б, для улучшения характеристик ЛАМ целесообразно увеличивать ширину шины $t$ только до определенного предела, т. е. до оптимального значения $t$, которое тем больше, чем меньше $\varepsilon_{0}$.

Полученные результаты позволяют более строго оценивать совместное влияние продольного и поперечного краевых эффектов на параметры ЛАМ.

\title{
ЛИТЕРАТУРА
}

1. Вольдек А., Скря бина Г. Теория линейной асинхпонной машины с учетом продольного и попепечного краевых эффектов. - Изв. АН ЭССР, Физ. Матем., 1978 , т. 27. № 2, с. 202-210.

2. Вольдек А. И. Индукционные магнитогидродинамические машины с жидкометаллическим рабочим телом. Л.. 1970.

3. В ольдек А. И., Т олвин ск я Е. В. Метод расчета характернстик линейных и дуговых машин с учетом влияния продольного краевого эффекта. - Marнитная гидродинамика, 1971 , № 1, с. 84-90.

4. Л ан с Дж. Н. Численные методы для быстродействующих вычислительных машин. М., 1962.

Ленинградский политехнический институт им. М. Н. Калинина

Поступила в педакцию 29/III 1977

\section{A. VOLDEK , A. IVANOV, G. SKRJABINA}

\section{PIKI- JA RIST-Å̈REEFEKTI ARVESTAV LINEAARSE ASONKROONMASINA INTEGRAALKARAKTERISTIKUTE ARVUTUS}

Artiklis on esitatud valemid lineaarse asünkroonmootori ekvivalentskeemide parameetrite ia mehaaniliste karakteristikute arvutamiseks, kusiuures on arvestatud piki- ja rist-ääreefekti, ning ülalnimetatud suuruste numbrilised väärtused.

\author{
A. VOLDEK|, A. IVANOV, G. SKRYABINA
}

\section{THE CHARACTERISTICS OF LINEAR INDUCTION MACHINE WITH END AND TRANSVERSE EDGE EFFECTS}

The authors present analytic equations for the equivalent impedance and mechanical characteristics of the linear induction machine with longitudinal end and transverse edge effects, using two-dimensional theorv of that machine. Numerical results for a particular motor are obtained and discussed. 